\title{
INFLUENCE OF THE CPRMETER ON ANGULAR POSITION OF ELBOWS AND GENERATED FORCES DURING CARDIOPULMONARY RESUSCITATION
}

\author{
KAROLINA KOPACZ ${ }^{1}$, MAGDALENA FRONCZEK-WOJCIECHOWSKA ${ }^{1}$, ANNA JAŹWIŃSKA ${ }^{2}$, \\ GIANLUCA PADULA ${ }^{1}$, DARIUSZ NOWAK ${ }^{3}$, and TOMASZ GASZYŃSKI ${ }^{2}$
}

Medical University of Lodz, Łódź, Poland

1 "DynamoLab" Academic Laboratory of Movement and Human Physical Performance

${ }^{2}$ Department of Emergency Medicine and Disaster Medicine

${ }^{3}$ Department of Clinical Physiology

\begin{abstract}
Objectives: It is commonly known that ergonomics in emergency medical services (EMS) is very important. Emergency medical services workers are exposed to different conditions and they should perform a variety of tasks. Material and Methods: The aim of the work has been to analyze the angular position of elbows and forces generated by the upper limbs during cardiopulmonary resuscitation with and without the CPRmeter based on feedback technology. Ten male paramedics and 10 male non-paramedics, in a kneeling position, performed cardiopulmonary resuscitation (CPR) on an Ambu Megacode manikin placed on the ground. Measurements were taken after 1 min and 4 min following the beginning of the trial. The angular position of the elbows was evaluated with a BTS Smart DX 7000 motion capture system. Kistler platforms 9286BA were used for measuring forces. Results: In the paramedic group, one statistically significant difference was observed in the mean difference between maximal and minimal right elbow angle in the 1st min without the device vs. the mean difference in the 4th min without the device. In the paramedic group, a $25 \%$ force decrease was observed after 4 min of resuscitation in trials without the CPRmeter in comparison to the 1st min. In trials with the CPRmeter, the force parameters were similar in the 1st and 4th min and more stable. No statistically significant differences were noticed in the control group. Conclusions: The CPRmeter has influence on the magnitude of the forces applied by the upper limbs and on the optimization of the rescuer effort during cardiopulmonary resuscitation. The CPRmeter had no influence on the position of the upper part of the kinematic chain. Int J Occup Med Environ Health 2017;30(6):909-916
\end{abstract}

Key words:

Emergency medicine, Paramedics, Cardiopulmonary resuscitation, Force, Objective movement analysis, CPRmeter

\section{INTRODUCTION}

The 2015 American Heart Association (AHA) Guidelines for Cardiopulmonary Resuscitation (CPR) and the Emergency Cardiovascular Care (ECC) emphasize that early, high-quality chest compression plays a key role in resuscitation after cardiac arrest [1]. It is also important to continue research with the purpose of improving the quality of resuscitation and to update the knowledge of rescuers.

Received: July 22, 2016. Accepted: November 9, 2016.

Corresponding author: K. Kopacz, Medical University of Lodz, "DynamoLab" Academic Laboratory of Movement and Human Physical Performance, Pomorska 251, 92-216 Łódź, Poland (e-mail: karolina.kopacz@umed.lodz.pl). 
Ashton et al. [2] and Lockley and Nolan [3] also emphasized the need to apply evidence-based research to Emergency Medicine.

The basic life support (BLS) sequence given in the AHA Guidelines for CPR and ECC has changed from A-B-C (Airway, Breathing, Chest compressions) to C-A-B (Chest compressions, Airway, Breathing) for adults, children, and infants (excluding the newly born). According to the authors of the 2015 AHA Guidelines for CPR and ECC, this change has been necessary as the vast majority of cardiac arrests occur in the case of adult patients where it is vital to decrease the delay in circulation. The rescuer should also use CPR of a high quality with regard to the rate and depth of chest compression, allowing for complete chest recoil after each compression, minimizing interruptions in compressions, and avoiding excessive ventilation.

It is commonly known that ergonomics in emergency medical services (EMS) is very important. Emergency medical services workers are exposed to different conditions and they should perform a variety of tasks [4]. Some studies have discussed the posture of the rescuer, which may influence the chest compression depth and rate $[5,6]$. The 2015 CPR guidelines recommend that the position of the resuscitation provider should be vertically above the chest of the victim, with straight arms [7]. However, the upper limb must be in the correct configuration to perform high-quality chest compression.

Other studies have demonstrated the importance of attaining correct hand position during CPR [8,9]. The CPRmeter is a small device which gives a dynamic real-time feedback related to depth and rate of chest wall movement during compression. It also detects whether the patient's chest is allowed to fully release between compressions. The device has 2 sensors: one measuring acceleration and the other measuring force. A microprocessor located in the middle of the device continuously measures both parameters during each compression. An implemented algorithm converts the collected data into specific information for the rescuer.
As much recent research [10,11] has addressed the potential of using devices to improve the quality of CPR, the primary aim of this work has been to investigate the influence of the CPRmeter on the angular position of the elbows during cardiopulmonary resuscitation. Achieving the correct hand position, to direct forces on a perpendicular direction to the chest, has influence on the magnitude of the forces applied and the efficacy of the procedure. The second aim of the work has been to verify whether the feedback of the CPRmeter has influence on the forces generated by the upper limbs.

\section{MATERIAL AND METHODS}

\section{Study group}

The study involved 20 adult men divided into 2 groups: in the paramedic group - 10 students of the 5th year of Public Health with specialty in Emergency Medicine (mean age: 23 years old; mean height: $1.82 \mathrm{~m}$; mean body mass index (BMI): $22 \mathrm{~kg} / \mathrm{m}^{2}$ ), and in the control group - 10 students who were not paramedics but trained once (not regularly) to provide CPR (mean age: 24 years old; mean height: 1.80 m; mean BMI: $23 \mathrm{~kg} / \mathrm{m}^{2}$ ). The inclusion criteria were the written informed consent and declaration to comply with instructions related to participation in the study. The exclusion criteria included ingestion of any amount of alcohol, tea or coffee within $12 \mathrm{~h}$ preceding the trial, and consumption of any meal within $60 \mathrm{~min}$ before the test. The study was undertaken at the "DynamoLab" Academic Laboratory of Movement and Human Physical Performance of the Medical University of Lodz, Poland.

\section{Study protocol}

Paramedics and controls were examined during resuscitation in kneeling position, performed with an Ambu Megacode manikin placed on the force platform. Feedback about the depth, release, and rate of chest compressions throughout the CPR procedure was provided by 
a CPRmeter with Q-CPR technology attached to the manikin chest with adhesive plaster (Laerdal Medical Limited, UK). Readings were taken after 1 min and 4 min following the beginning of the resuscitation procedure.

Each subject performed the resuscitation bout twice: once with and once without the CPRmeter. Randomization of the resuscitation sequence was applied. Each subject was asked to draw one card from a box containing 10 cards -5 green and 5 red ones. In the case of choosing a green card, the subject was asked to perform 5-minute resuscitation with the CPRmeter and, after 1-hour break, he was asked to perform the resuscitation again but without the device. Choosing a red card obliged the subject to perform 5-minute resuscitation with inverted order of succession. Only drinking of mineral water was allowed during the breake between resuscitation bouts. All subjects had no previous experience in using the CPRmeter.

The research protocol was approved by the Bioethical Committee of the Medical University of Lodz (process number RNN/22/14/KB; 14 January 2014).

\section{Assessment of angular position and forces}

The angular position of the elbows was evaluated with a BTS Smart DX 7000 motion capture system (BTS Bioengineering, Italy). Reflective markers were placed on the bony structures of the upper limb: left and right acromion, left and right lateral epicondyle of the humerus, left and right styloid process of the radius (Photo 1). Left elbow angle was marked with 3 markers: left acromion, left lateral epicondyle of the humerus, left styloid process of the radius. Right elbow angle was marked with 3 markers: right acromion, right lateral epicondyle of the humerus, right styloid process of the radius. Kistler platforms 9286BA (Kistler, USA) were used for measuring ground reaction force with a $400 \mathrm{~Hz}$ frequency. Data processing was performed with the use of SMARTtracker version 1.10.451.0, SMARTanalyzer - version 1.10.0225, Microsoft Excel 2010 and Statistica 10.

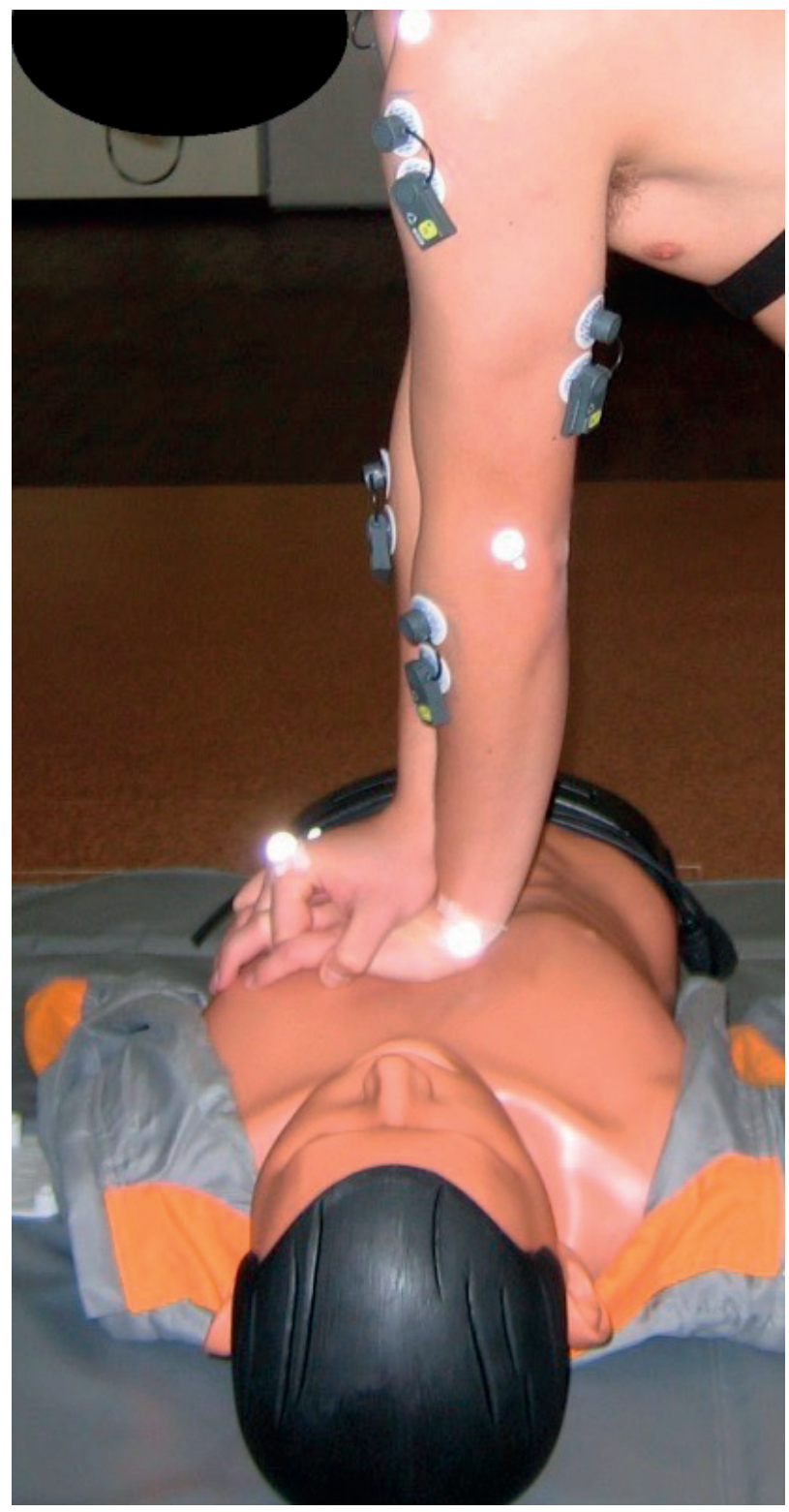

Photo 1. Ambu Megacode manikin cardiopulmonary resuscitation (CPR) - placement of reflective markers on the upper limb of the study participant during evaluation of angular position of the elbows

\section{Statistical analysis}

Results are expressed as a mean and standard deviation. Data distribution was tested with the Shapiro-Wilk test of normality. According to the data distribution, the following tests were used: paired t-test or Wilcoxon test for within group comparisons (dependent variables) and 
unpaired t-test or the Mann-Whitney $\mathrm{U}$ test for between groups comparisons (independent variables). The BrownForsythe test for analysis of the equality of the group variances was used prior to the application of the unpaired t-test and, if variances were unequal, Welch's t-test was used instead of the standard t-test. A p value of $<0.05$ was considered significant.

\section{RESULTS}

\section{The angular position of elbows}

It was noticeable that mean values related to angular position of elbows were similar during resuscitation with and without the CPRmeter in both groups (Table 1). The only exception in the control group was the parameter related to the mean left elbow angle in the 4th min, significantly different comparing resuscitations with and without the device. In both groups investigated, the biggest difference between minimum and maximum elbow angular position concerned left elbow angle in the 1st min of resuscitation without the device. The smallest difference between minimum and maximum elbow angular position regarded the right elbow angle in the 4th min of resuscitation without the device. Mean elbow angular values related to resuscitation with the CPRmeter were similar in the 1st and 4th min in both groups.

No statistically significant results were found in mean angles within the paramedic group. However, there was one statistically significant difference within the control group related to the mean left elbow angle in the 4th min with the device and without the device $(p=0.0355)$. In comparison to the paramedics, the control group showed no statistically significant differences in mean angles.

There were no statistically significant differences of mean difference between maximal and minimal angle within the paramedic group. Only one statistically significant difference was observed in the paramedic group and it was

Table 1. Angular position of elbows during cardiopulmonary resuscitation (CPR)* performed with and without CPRmeter by paramedics and non-paramedics (control group)

\begin{tabular}{|c|c|c|c|c|c|c|c|c|c|}
\hline \multirow{4}{*}{ Variable } & \multicolumn{8}{|c|}{$\begin{array}{c}\text { Angular value } \\
{\left[^{\circ}\right]} \\
(\mathrm{M} \pm \mathrm{SD})\end{array}$} & \multirow{4}{*}{$\mathrm{p}^{* *}$} \\
\hline & \multicolumn{4}{|c|}{ paramedic group } & \multicolumn{4}{|c|}{ control group } & \\
\hline & \multicolumn{2}{|c|}{ without device } & \multicolumn{2}{|c|}{ with device } & \multicolumn{2}{|c|}{ without device } & \multicolumn{2}{|c|}{ with device } & \\
\hline & $1 \mathrm{st} \min$ & 4th $\min$ & 1st $\min$ & 4th min & 1st $\min$ & 4th $\min$ & 1st min & 4th min & \\
\hline Right elbow angle & $163 \pm 8$ & $163 \pm 7$ & $162 \pm 9$ & $162 \pm 8$ & $164 \pm 5$ & $164 \pm 5$ & $164 \pm 4$ & $162 \pm 4$ & n.s. \\
\hline Left elbow angle & $162 \pm 7$ & $161 \pm 7$ & $163 \pm 6$ & $161 \pm 6$ & $161 \pm 6$ & $161 \pm 6^{c}$ & $161 \pm 7$ & $157 \pm 9^{c}$ & $\mathrm{c}$ \\
\hline $\begin{array}{l}\text { Difference between maximal } \\
\text { and minimal right elbow angle }\end{array}$ & $15 \pm 7^{7, b}$ & $12 \pm 4^{b}$ & $14 \pm 8$ & $14 \pm 7$ & $10 \pm 3^{a}$ & $9 \pm 3^{c}$ & $11 \pm 7$ & $11 \pm 4^{c}$ & $a, b, c$ \\
\hline $\begin{array}{l}\text { Difference between maximal } \\
\text { and minimal left elbow angle }\end{array}$ & $17 \pm 8$ & $13 \pm 5$ & $14 \pm 6$ & $15 \pm 7$ & $14 \pm 4$ & $12 \pm 4$ & $16 \pm 6$ & $16 \pm 6$ & n.s. \\
\hline
\end{tabular}

* The CPR was performed in a kneeling position on an Ambu Megacode manikin placed on the ground with or without the CPRmeter. Measurements evaluated with a BTS Smart DX 7000 motion capture system were taken after 1 min and 4 min following the beginning of the trial.

** Statistically significance differences $-\mathrm{p}<0.05$ :

a in the 1st min without the device - paramedic vs. control group;

${ }^{b} 1$ st $\min$ vs. 4th min without the device in the paramedic group;

${ }^{c}$ in the 4th min with vs. without the device in the control group.

n.s. - not statistically significant. 
related to the mean difference between maximal and minimal right elbow angle in the 1 st min vs. the 4th min without the device ( $p=0.0366)$. In the control group, there was one statistically significant difference in relation to the mean difference between maximal and minimal right elbow angle in the 4th min with and without the device ( $p=0.0097)$. Comparing the paramedic and control group, a statistically significant difference was observed in the mean difference between maximal and minimal right elbow angle in the 1st min without the device ( $\mathrm{p}=0.0467)$.

\section{Force parameters}

During resuscitation, in the study performed, subjects from the control group used more force then participants from the paramedic group in all cases (Table 2).

There were no statistically significant differences for the mean force in the control group. However, a statistical difference was observed in the paramedic group between the 4th min with and without the device ( $\mathrm{p}=0.0390)$. Moreover, in the case of the paramedic group, statistically significant differences were observed between: 1 st min without the device vs. 4th min without the device $(p=0.0050)$; 1 st $\mathrm{min}$ without device vs. 1st min with the device $(p=0.0366)$.
A statistically significant difference between groups was observed for the mean force in the 4th min of the CPR with the device ( $p=0.0015)$. Moreover, another statistically significant difference was noticed for the mean force in the 4th min of the CPR without the device $(p=0.0003)$. There were also statistically significant differences between paramedics and control group in the following parameters: mean force in the 1 st min of the $\mathrm{CPR}$ without the device $(p=0.0257)$; mean force in the 1 st min of CPR with the device $(\mathrm{p}=0.0022)$.

\section{DISCUSSION}

Some researchers have described physical demands associated with the EMS worker job tasks. Paramedics often have to turn the patients, transport them down the stairs, lift them, perform cardiopulmonary resuscitation, even if the external conditions are poor or there is a limited number of personnel $[4,12]$. That is why there is a need to use evidencebased medicine to improve the ergonomics in paramedics.

As many poorly-trained rescuers are known to perform poor quality resuscitation [13,14], various methods for improving the CPR quality, such as audio and visual feedback systems, have been developed. You et al. [15] have used a Heart-

Table 2. Force used during cardiopulmonary resuscitation $(\mathrm{CPR})^{*}$ performed by paramedics and non-paramedics (control group)

\begin{tabular}{|c|c|c|c|c|c|}
\hline \multirow{3}{*}{ Study group } & \multicolumn{4}{|c|}{$\begin{array}{c}\text { Force } \\
{[\mathrm{N}]} \\
(\mathrm{M} \pm \mathrm{SD})\end{array}$} & \multirow{3}{*}{$\mathrm{p}^{* *}$} \\
\hline & \multicolumn{2}{|c|}{ without device } & \multicolumn{2}{|c|}{ with device } & \\
\hline & $1 \mathrm{st} \min$ & 4th $\min$ & $1 \mathrm{st} \min$ & 4th $\min$ & \\
\hline Paramedic & $194.51 \pm 58.34^{\mathrm{a}, \mathrm{c}}$ & $144.68 \pm 29.22^{c}$ & $176.28 \pm 69.15^{\mathrm{a}}$ & $172.09 \pm 41.28^{b}$ & $\mathrm{a}, \mathrm{b}, \mathrm{c}$ \\
\hline Control & $255.37 \pm 66.35^{\mathrm{ad} d}$ & $242.53 \pm 57.25^{\mathrm{e}}$ & $281.93 \pm 57.76^{\mathrm{a}, \mathrm{d}}$ & $263.62 \pm 65.53^{\mathrm{b}, \mathrm{e}}$ & $\mathrm{a}, \mathrm{b}, \mathrm{d}, \mathrm{e}$ \\
\hline
\end{tabular}

* The CPR was performed in a kneeling position on an Ambu Megacode manikin placed on the ground with or without the CPRmeter. Measurements evaluated with Kistler platforms 9286BA were taken after 1 min and 4 min from the beginning of the trial.

** Statistically significance differences:

${ }^{a}$ paramedic vs. control group in the 1st min with and without the device;

${ }^{\mathrm{b}}$ paramedic vs. control group in the 4th min with the device;

c 1 st min vs. 4th min in paramedic group without the device;

${ }^{d}$ in the 1st min with vs. without the device in the control group;

${ }^{\mathrm{e}}$ in the 4 th min with vs. without the device in the control group. 
Saver Sticker to ensure that correct hand position is maintained during chest compressions as well as compression rate and depth with the use of video cameras. The results have confirmed that using a HeartSaver Sticker significantly improve correct hand placement. Heidenreich et al. [16] have compared standard vs. hands-only cardiopulmonary resuscitation. They have shown that $2 \mathrm{~min}$ after the initiation of hand-only cardiopulmonary resuscitation by young, healthy medical students, the rate of compression drop. That is why supporting methods based on visual feedback devices like the CPRmeter, among others, might be helpful.

Moreover, incorrect upper limb position during chest compression may influence the rescuer workload $[17,18]$. This will, in turn, influence the rate of fatigue and negatively affect the quality of the CPR. Hence, this study has been performed to evaluate the benefit of the CPRmeter in maintaining correct elbow position during chest compression and to investigate whether this device causes loss of forces generated by the subjects during resuscitation.

Studies have also reported a remarkable problem of fatigue during the CPR. The quality of chest compression declines more rapidly over time with the increased number of chest compressions [19,20]. Ochoa et al. [21] have shown that although fatigue occurs within $60 \mathrm{~s}$ following the beginning of the CPR, resuscitation providers may not recognize that until $2 \mathrm{~min}$ later. The results of this study have highlighted a significant difference in both groups observed in the mean values for maximal and minimal right elbow angle in the 1st and 4th min when the CPRmeter is not being used. The lack of any other significant differences has shown that the elbow angle has been stable both with and without the CPRmeter.

The movement analysis is commonly used in resuscitation research representing an important clinical evaluation method [22-24]. Studies have emphasized the role of hand position to obtain good CPR results.

However, only few articles have evaluated the elbow angle of the provider during CPR or the role of the upper part of the kinematic chain during the correct CPR. By using a motion analysis system to define patterns of joint movement, evaluate the kinematic profile, forces, depths, and frequencies during standard cardiopulmonary resuscitation vs. over-the-head resuscitation, Chi et al. [5] have demonstrated that no significant differences in elbow range of motion have been obtained using either method. In a second article, Chi et al. [25] used the 3-dimensional motion analysis to record the shoulder angle of the rescuer in different manikin positions. The results revealed that when the manikin was placed on a table $63 \mathrm{~cm}$ in height, the upper limb was more extended than when the manikin was lying on the floor.

Our research investigated the effect of using the CPRmeter on the elbows angular position demonstrating that the rescuer could maintain the upper extremities with elbows extended for 5 min after chest compression started, whether the CPRmeter was used or not. These results showed that the elbows position was almost the same during resuscitation without and with this device, which might have suggested that the students examined had been well trained and the quality of CPR performed by them was of a high standard. Other researchers highlighted the meaning of force generated by upper limbs during resuscitation. Tomlinson showed the correlation between the compression force and compression depth [26]. The study has demonstrated that adequate chest compression depth may be achieved by applying $50 \mathrm{~kg}$ force to the sternum. Beesems et al. [27] observed that there was a tendency to decreasing force compression over time during resuscitation, similar in male and female subjects.

Our results demonstrated that subjects from the control group put more strength during cardiopulmonary resuscitation than the paramedics. Except that, the former used more force during resuscitation with the device than without it. An explanation of the result may be a possible anxiety of sliding off the device from the chest, even though the device was attached by the adhesive plaster 
to the thorax of the manikin. In the paramedic group, we observed $25 \%$ loss of force after 4 min of resuscitation without the CPRmeter in comparison to the 1st min. In trials with the CPRmeter, instead, the force parameters were similar in the 1st and the 4th min and more stable.

According to this study, the CPRmeter seems a good equipment for the CPR trainings and could help optimize the rescuer effort during the CPR. Our work showed that the paramedic group had better sense of force used during resuscitation and generated less force in comparison to the control group. Moreover, during trials with the CPRmeter, the force parameters generated by them were more stable. This has certainly a positive impact on the optimization of the rescuer effort.

Force data related to the control group has shown higher values than paramedics during the $\mathrm{CPR}$, which may result in a faster fatigue and make the CPR ineffective. In this context, optimal depth and force have influence on the rescuer effort optimization, which leads to the decreased number of complications, e.g., broken ribs.

\section{CONCLUSIONS}

We have found that the CPRmeter has influence on the magnitude of the forces applied by the upper limbs and on the optimization of the rescuer effort during cardiopulmonary resuscitation.

The CPRmeter has no influence on the position of the upper part of the kinematic chain.

It is to be hoped that real-time feedback devices may be an integral part in simulations or improve outcomes in cardiac arrest.

\section{REFERENCES}

1. American Heart Association. Highlights of the 2015 American Heart Association guidelines update for CPR and ECC. Dallas: The Association; 2015.

2. Ashton A, McCluskey A, Gwinnutt CL, Keenan AM. Effect of rescuer fatigue on performance of continuous external chest compression over 3 min. Resuscitation. 2002;55:151-5, https://doi.org/10.1016/S0300-9572(02)00168-5.

3. Lockey AS, Nolan JP. Cardiopulmonary resuscitation in adults. Revised guidelines are more evidence based. BMJ. 2001;323:819-20, https://doi.org/10.1136/bmj.323.7317.819.

4. Conrad KM, Reichelt PA, Lavender SA, Gacki-Smith J, Hattle S. Designing ergonomic interventions for EMS workers: Concept generation of patient-handling devices. Appl Ergon. 2008;39:792-802, https://doi.org/10.1016/j.apergo.2007.12.001.

5. Chi CH, Tsou JY, Su FC. Comparison of chest compression kinematics associated with over-the-head and standard cardiopulmonary resuscitation. Am J Emerg Med. 2009;27:1112-6, https://doi.org/10.1016/j.ajem.2008.08.029.

6. Larsen PD, Perrin K, Galletly DC. Patterns of external chest compression. Resuscitation. 2002;53:281-7, https:// doi.org/10.1016/S0300-9572(02)00026-6.

7. Resuscitation Council. The resuscitation guidelines 2010. London: The Council; 2010.

8. Diószeghy C, Kiss D, Fritúz G, Székely G, Elo G. Comparison of effects of different hand positions during cardiopulmonary resuscitation. Resuscitation. 2005;66:297-301, https://doi.org/10.1016/j.resuscitation.2005.03.010.

9. Shin J, Rhee JE, Kim K. Is the inter-nipple line the correct hand position for effective chest compression in adult cardiopulmonary resuscitation? Resuscitation. 2007;75:305-10, https://doi.org/10.1016/j.resuscitation.2007.05.003.

10. Gruber J, Stumpf D, Zapletal B, Neuhold S, Fischer H. Real-time feedback systems in CPR. Curr Anaesth Crit Care. 2012;2:287-94, https://doi.org/10.1016/j.tacc.2012.09.004.

11. Kirkbright S, Finn J, Tohira H, Bremner A, Jacobs I, Celenza A. Audiovisual feedback device use by health care professionals during CPR: A systematic review and meta-analysis of randomised and non-randomised trials. Resuscitation. 2014;85(4):460-71, https://doi.org/10.1016/j.resuscita tion.2013.12.012.

12. Garrison HG. Keeping rescuers safe. Ann Emerg Med. 2002;40:633-5, https://doi.org/10.1067/mem.2002.129940. 
13. Handley AJ. Teaching hand placement for chest compression - A simpler technique. Resuscitation. 2002;53:29-36, https://doi.org/10.1016/S0300-9572(01)00506-8.

14. Chamberlain D, Smith A, Colquhoun M, Handley AJ, Kern KB, Woolard M. Randomised controlled trials of staged teaching for basic life support: 2. Comparison of CPR performance and skill retention using either staged instruction or conventional training. Resuscitation. 2001;50:27-37, https://doi.org/10.1016/S0300-9572(01)00342-2.

15. You JS, Chung SP, Park JY, Park S, Chung TN, Park I, et al. The utility of the HeartSaver Sticker for maintaining correct hand position during chest compressions. J Emerg Med. 2012;43:184-9, https://doi.org/10.1016/j.jemermed.2011.05.060.

16. Heidenreich JW, Bonner A, Sanders AB. Rescuer fatigue in the elderly: Standard vs. hands-only CPR. J Emerg Med. 2012;42:88-92, https://doi.org/10.1016/j.jemermed.20 10.05.019.

17. Hong CK, Park SO, Jeong HH, Kim JH, Lee NK, Lee KY, et al. The most effective rescuer's position for cardiopulmonary resuscitation provided to patients on beds: A randomized, controlled, crossover mannequin study. J Emerg Med. 2014;46:643-9, https://doi.org/10.1016/j.jemermed.2013.08.085.

18. Yang Z, Li H, Yu T, Chen C, Xu J, Chu Y, et al. Quality of chest compressions during compression-only CPR: A comparative analysis following the 2005 and 2010 American Heart Association guidelines. Am J Emerg Med. 2014;32: 50-4, https://doi.org/10.1016/j.ajem.2013.09.043.

19. Sugerman NT, Edelson DP, Leary M, Weidman EK, Herzberg DL, Vanden Hoek TL, et al. Rescuer fatigue during actual in-hospital cardiopulmonary resuscitation with audiovisual feedback: A prospective multicenter study. Resuscitation. 2009;80:981-4, https://doi.org/10.1016/j.resus citation.2009.06.002.

20. Bjørshol CA, Søreide E, Torsteinbø TH, Lexow K, Nilsen OB, Sunde K. Quality of chest compressions during $10 \mathrm{~min}$ of single-rescuer basic life support with different compression: Ventilation ratios in a manikin model. Resuscitation. 2008;77:95-100, https://doi.org/10.1016/j.resuscitation. 2007.11.009.

21. Ochoa FJ, Ramalle-Gómara E, Lisa V, Saralegui I. The effect of rescuer fatigue on the quality of chest compressions. Resuscitation. 1998;37:149-52, https://doi.org/10.1016/S0 300-9572(98)00057-4.

22. Hightower D, Thomas SH, Stone CK, Dunn K, March JA. Decay in quality of closed-chest compressions over time. Ann Emerg Med. 1995;26(3):300-3, https://doi.org/10.1016/ S0196-0644(95)70076-5.

23. Van Andel CJ, Wolterbeek N, Doorenbosch CA, Veeger DH, Harlaar J. Complete 3D kinematics of upper extremity functional tasks. Gait Posture. 2008;27:120-7, https://doi. org/10.1016/j.gaitpost.2007.03.002.

24. Chung WM, Yeung S, Chan WW, Lee R. Validity of VICON motion analysis system for upper limb kinematic measurement - A comparison study with inertial tracking Xsens system. Hong Kong Physiother J. 2011;29:97, https://doi.org/ 10.1016/j.hkpj.2011.08.015.

25. Chi CH, Tsou JY, Su FC. Effects of rescuer position on the kinematics of cardiopulmonary resuscitation (CPR) and the force of delivered compressions. Resuscitation. 2008;76: 69-75, https://doi.org/10.1016/j.resuscitation.2007.06.007.

26. Tomlinson AE, Nysaether J, Kramer-Johansen J, Steen PA, Dorph E. Compression force-depth relationship during out-of-hospital cardiopulmonary resuscitation. Resuscitation. 2007;72:364-70, https://doi.org/10.1016/j.resuscitation. 2006.07.017.

27. Beesems SG, Hardig BM, Nilsson A, Koster RW. Force and depth of mechanical chest compressions and their relation to chest height and gender in an out-of-hospital setting. Resuscitation. 2015;91:67-72, https://doi.org/10.1016/ j.resuscitation.2015.03.020.

This work is available in Open Access model and licensed under a Creative Commons Attribution-NonCommercial 3.0 Poland License - http://creativecommons.org/ licenses/by-nc/3.0/pl/deed.en. 